Chinese Journal of Organic Chemistry

\title{
常见大环自组装构建分子胶囊
}

郑点梁丹霞 手雪涛 陈仁尔 花江 周其忠*
(台州学院化学系台州 317000)

\begin{abstract}
摘要 分子胶囊以其独特的分子结构在控制药物释放、催化、材料科学和生物医药等领域具有潜在的应用价值. 分子 胶囊可由不同的分子模块通过共价键或非共价键构建. 着重描述了由常见的大环主体(环糊精、杯芳烃、杯吡咯、卟啉、 环三藜芦烯和葫芦腿)通过自组装形成的分子胶囊. 这些分子胶囊通过非共价键(氢键、静电作用、疏水作用及范德华力 等)连接, 使得分子胶囊具有可控的可调节的分子结构. 通过这些独特的分子结构, 分子胶囊可在溶液或固相中包裹各 种各样的有趣的客体.
\end{abstract}

关键词＼cjkstart超分子化学; 大环主体; 自组装; 分子胶囊

\section{Molecular Capsules Based on Common Macrocycles Self-Assembly}

\author{
Zheng, Rui $\quad$ Liang, Danxia $\quad$ Yu, Xuetao $\quad$ Chen, Rener \\ Jiang, Huajiang Zhou, Qizhong* \\ (Department of Chemistry, Taizhou University, Taizhou 317000)
}

\begin{abstract}
Molecular capsules are amazing and fascinating because of their unusual structure and nanospaces. These unique microenvironments and nano-structures ultimately ensure their potential for applications in materials science, biological science, drug delivery and catalysis. Molecular capsules could be constructed by covalent bonds or non-covalent bonds with many kinds of different components. This article describes the syntheses and properties of a library of diverse molecular capsules by non-covalent bonds with common macrocycles, which covers (i) cyclodextrins based systems, (ii) calix $[n]$ arenes based systems, (iii) calic[n]pyrroles based systems, (iv) porphyrins based systems, (v) cyclotriveratrylene (CTV) based systems, and finally (vi) cucurbit $[n]$ urils based systems. These building blocks afford molecular capsules based on the self-assembly of several units, which is primarily driven by the interplay of weak attractions that act over short distances between molecules, including hydrogen bonds, electrostatic interaction, aromatic $\pi$-stacking, van der Waal's interactions, etc. These versatile molecular capsules providing different nanospaces, some of which are controllable and adjustable, could encapsulate different kinds of interesting guests in solution or solid states.
\end{abstract}

Keywords supramolecular chemistry; macrocycle; self-assembly; molecular capsule

超分子化学是化学的一个重要的分支. 它是指通过 分子识别作用把单个或多个分子组装成分子聚集体. 分 子识别依赖于主客体分子的大小、形状和化学功能的互 补. 其通过分子间的非共价键作用力——氢键、静电作 用、疏水作用、 $\pi-\pi$ 堆积作用及范德华力等把基本的分 子构筑单元连接起来 ${ }^{[1]}$.

大环主体是这些构筑单元的重要组成部分. 每一代 大环主体的发现和发展都会极大地丰富超分子化学的 内容, 并推动超分子化学极快地发展. 从第一代的大环 主体冠醚被发现开始, 一系列的大环主体被设计合成出
来，如：环糊精、杯芳烃、杯吡咯、卟啉、环三菉芦烯 (CTV)和葫芦脲等. 以这些大环为基础模块, 通过分子 识别以分子间非共价键作用为基础构建了大量的有趣 的超分子体系，例如：轮烷、索烃、聚轮烷、分子笼和 分子胶囊等 ${ }^{[2 \sim 7]}$. 这些超分子体系被用于智能材料、传 感器、逻辑器件、载药体系和分子机器等领域. 其中通 过自组装设计和合成分子胶囊引起了越来越多的关注. 自组装是指一系列给定的基础单元通过非共价作用力 自发的形成结构高度有序的相对独立的一个超分子结 构. 例如: 两个或两个以上的分子通过分子间非共价的

*E-mail: qizhongchou@yahoo.com

Received September 3, 2012; revised September 20, 2012; published online October 11, 2012.

Project supported by the National Natural Science Foundation of China (No. 21172166) and the Natural Science Foundation of of Zhejiang Province (No. Y4100783).

国家自然科学基金(No. 21172166)和浙江省自然科学基金(No. Y4100783)资助项目. 
可逆的相互作用连接到一起形成一个球形内部有空腔 的超分子结构——这就是自组装形成的分子胶囊. 当 然, 分子胶囊也可以由共价键构建 ${ }^{[8]}$, 但是与共价键相 比, 由非共价键自组装形成的分子胶囊具有可控性和可 调节性. 这为制作可控的可调节的超分子体系提供了便 利. 分子胶囊有一个纳米空腔, 其可包裹合适的客体分 子, 把客体分子与周围环境隔离开来, 并赋予被包裹客 体独特的不同于被包裹前的性质, 例如: 客体的构象改 变 ${ }^{[9]}$ 、易变的客体变得稳定及客体的化学反应速度加 快 ${ }^{[10 ~ 17]}$ 等. 分子胶囊这一独特的性质使其在分子存储、 传感器、微反应器和生物医药等领域具有潜在应用价 值 $^{[18 ~ 24]}$. 本文着重介绍常见大环一一环糊精、杯芳烃、 杯吡咯、卟啉、CTV 和葫芦脲(如 Scheme 1 所示一些典 型的大环)或其衍生物通过自组装构建分子胶囊的方法 及性质.
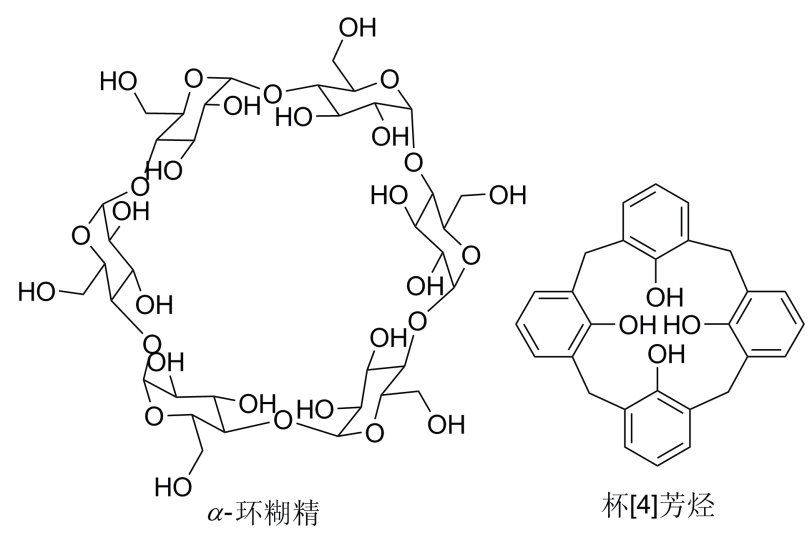

杯[4]芳烃

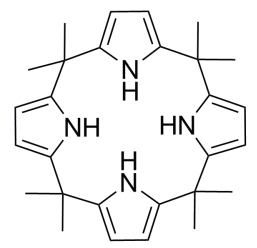

杯[4]吡咯

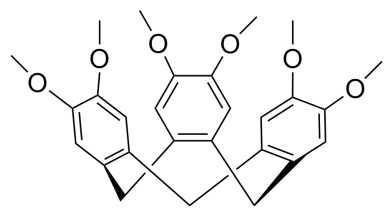

CTV

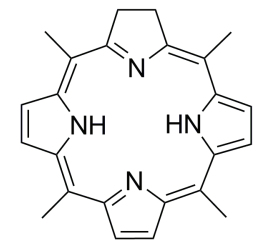

卟啉

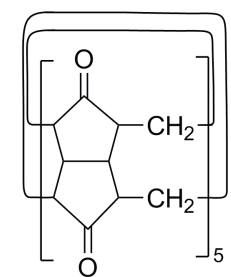

葫芦艮[5]
Scheme 1

\section{1 环糊精}

环糊精分子具有略呈雉形的中空圆筒状结构. 其可 作为基本的构筑单元构建分子胶囊. Jullien 等 ${ }^{[25]}$ 将 $\beta$-环 糊精分别用氨基和羧基衍生化, 制备了两种带有相反电
荷的 $\beta$-环糊精衍生物. 在 $50 \mathrm{mmol} / \mathrm{L}$ 的 $\mathrm{KCl}$ 水溶液中, 这两种 $\beta$-环糊精衍生物可通过静电作用自组装成分子 胶囊 (Eq. 1). 分子胶囊的结构通过动态核磁表征 (dynamic ${ }^{1} \mathrm{H}$ NMR). 脉冲梯度自旋回波核磁(PGSE ${ }^{1} \mathrm{H}$ NMR)实验结果表明: 这两种发生相互作用的衍生物在 二聚时的分子间距离决定了自组装体一一分子胶囊的 生成. 这一距离的范围为 $0.4 \sim 1.1 \mathrm{~nm}$. 当两者间的距离 为 $0.6 \mathrm{~nm}$ 时, 两者最有可能组装成分子胶囊.

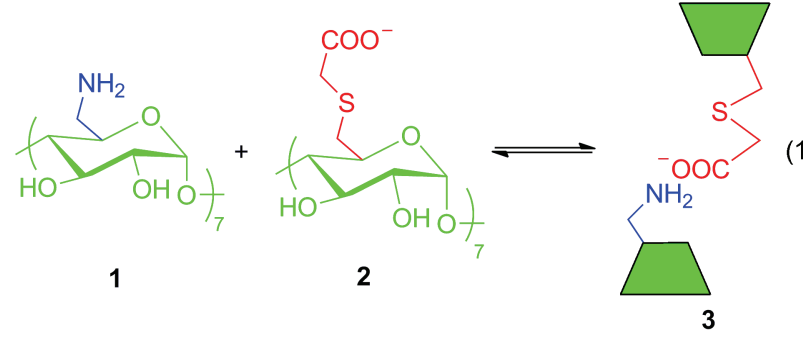

$\mathrm{Nau}$ 等 ${ }^{[26]}$ 发现 $\beta$-环糊精可在水溶液中与镜像异构 的手型客体组装形成对映异构的分子胶囊. 这一 $2: 1$ 的超分子复合体系会从水中沉淀出来, 从而达到对应异 构体手性拆分的目的(Scheme 2). $\beta$-环糊精与镜像异构 的客体组装成 $1: 1$ 和 $2: 1$ 的复合物的络合常数是不同 的. 这一差异导致对映异构体(客体)与 $\beta$-环糊精结合后 在水中的溶解性降低, 从而从水中沉淀下来. 与左消旋 的客体相比, 右消旋的客体与 $\beta$-环糊精组装形成的分子 胶囊后更易从水中沉淀下来. 因此, 沉淀中右消旋客体 的含量高于左消旋的客体. 通过诱导圆二色谱研究发 现，对映体过量率为 $(30 \pm 3) \%$. 其与左消旋客体和右消 旋客体分别与 $\beta$-环糊精形成的 $1: 1$ 和 $2: 1$ 的复合物的 络合常数有关.
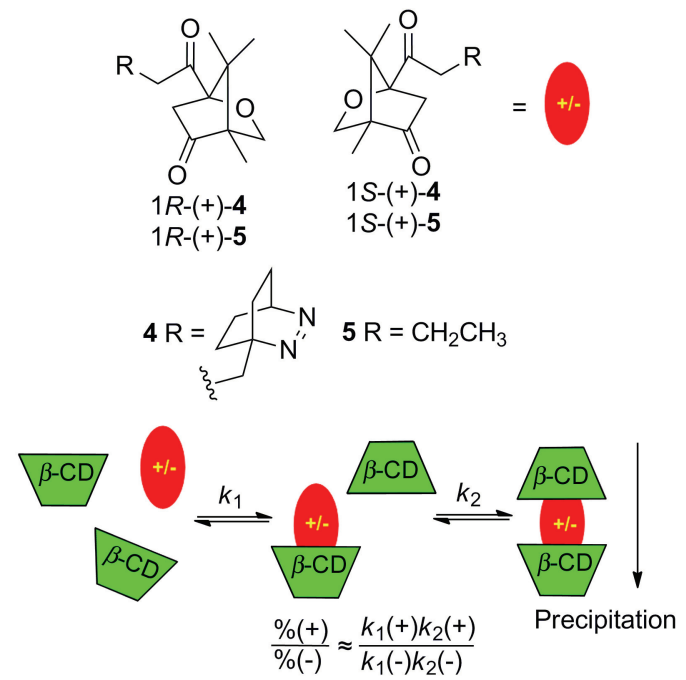

Scheme 2

$\alpha$-环糊精也可用于对映异构体的手性识别. Ejchart 等 ${ }^{[27]}$ 发现 $\alpha$-环糊精可与樟脑对映异构体在水中以 $2: 1$ 
的方式组装成分子胶囊, 并研究了客体分子在胶囊中的 定向问题. 通过核磁共振分析发现: 与左消旋的対映体 相比，右消旋的対映体上的一些甲基在胶囊中转动更自 由. 这使得右消旋的客体与 $\alpha$-环糊精的结合能力弱于左 消旋的客体.

除此之外, 环糊精形成的胶囊还可用于控制释放. Toro-Sánchez 等 ${ }^{[28]}$ 发现 $\beta$-环糊精分子胶囊可以在水与乙 醇的混合相中包裹百里香精油. 红外色谱和核磁共振研 究发现氢键和疏水作用是使得 $\beta$-环糊精分子胶囊与百 里香精油络合的驱动力. 单独存在的百里香精油极易挥 发, 但是其被 $\beta$-环糊精分子胶囊包裹后, 可使其具有抗 真菌的挥发物的释放变得可控.

\section{2 杯芳烃}

杯芳烃分子是上宽下窄、内有空穴的 “杯状” 结构, 并且其极易通过衍生化获得特定的功能. 这一特性使得 杯芳烃和其衍生物天然的作为基本骨架构建分子胶囊. 目前，用于组装分子胶囊的杯芳烃主要有以下几类：磺 酸化的杯芳烃、焦棓酚形杯芳烃和雷锁酚形杯芳烃等.

\section{1 磺酸化的杯芳烃}

对位磺酸盐杯芳烃带有多电荷具有极好的水溶性 (Scheme 3), 常被用于超分子体系的自组装. 它在分子 胶囊的构建中也扮演了重要角色.

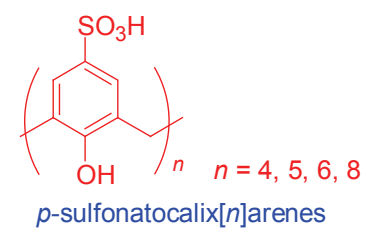

\section{Scheme 3}

Raston 等 ${ }^{[30]}$ 利用对磺酸杯[4]芳烃、4,13-二氮-18冠-6 和镧系金属离子在水溶液中自组装形成分子胶囊, 并得到其晶体结构(Scheme 4). 在晶体结构中, 质子化 的 4,13-二氮-18-冠-6 通过氢键和两个水分子连接. 它们 被包裹在两个对磺酸盐杯[4]芳烃形成的分子胶囊中; 两个镧系金属离子和对磺酸盐杯 $[4]$ 芳烃上的两个磺酸 基通过氢键连接.

Raston 等 ${ }^{[31]}$ 利用对磺酸杯 [4]芳烃的钠盐和三氯化 钇或三氯化铕在的水溶液中组装成分子胶囊 (Scheme 5). 其晶体结构显示, 两个水合的钠原子分别和两个对 磺酸杯[4]芳烃上的磺酸基相接, 把两个对磺酸杯[4]芳 烃桥连起来. 但是两个对磺酸杯 [4]芳烃并没有以 “头对 头” 的形式完全对齐, 而是形成了一个扭曲的分子胶囊. 在分子胶囊的中间包裹了两个水分子.
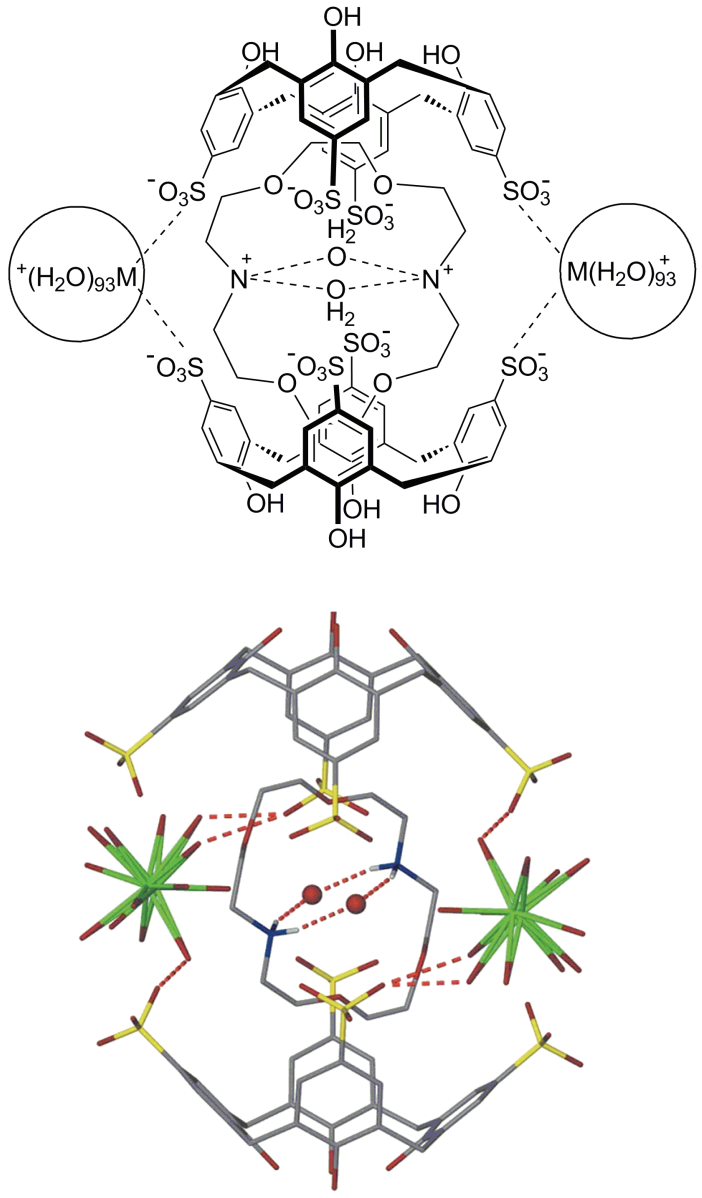

Scheme 4

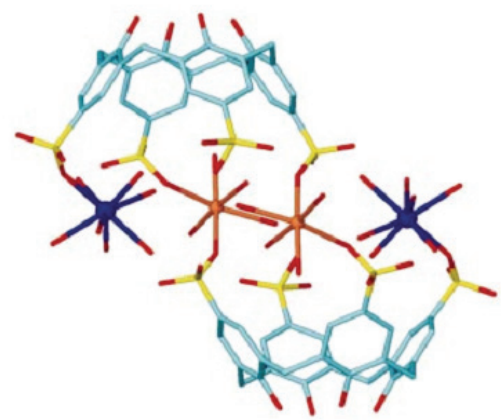

Scheme 5

Raston 等 ${ }^{[32]}$ 利用硫酸处理杯[5]芳烃得到了对磺酸 杯[5]芳烃组装成的分子胶囊. 此分子胶囊由对磺酸杯 [5]芳烃上的羧基以氢键稳定，胶囊中间以氢键固定了 两个硫酸分子.

\section{2 焦棓酚形杯芳烃}

虽然焦棓酚形杯芳烃(如 Scheme 6 所示的焦棓酚形 杯[4]芳烃)很早就被合成出来，但是直到现在其仍能表 现出非凡的性质，并被大量应用. 它含有带 3 个羟基的 重复单元，是很好的氢键给体和受体. 因此，焦棓酚形 
杯芳烃及其衍生物被大量的用于组装分子胶囊.<smiles>Oc1ccccc1Cc1cc(Cc2cc(Cc3cc(Cc4cc(Cc5cc(O)c(O)c(O)c5O)c(O)c(O)c4O)c(O)c(O)c3O)c(O)c(O)c2O)c(O)c(O)c1O</smiles>

pyrogallol[4]arene

\section{Scheme 6}

Purse 等 ${ }^{[33]}$ 利用焦棓酚杯 [4]芳烃的衍生物组装成分 子胶囊包裹萠和 $\mathrm{CDCl}_{3}$ (Scheme 7). 他们将等量的焦棓 酚杯 [4]芳烃和萠用研针研磨, 然后加入 10 倍量的固体 石蜡, 加热到 $100{ }^{\circ} \mathrm{C}$. 石蜡融化, 所得固体为产物, 加 入 $\mathrm{CDCl}_{3}$, 经分析检测, 得到的是由 6 个焦棓酚杯 [4]芳 烃组成的分子胶囊, 并在其中包裹了 3 个萠分子; 此分 子胶囊是动力学稳定的, 但不是热力学稳定的. 如果将 此包裹了萠的分子胶囊在 $\mathrm{CDCl}_{3}$ 中 $70{ }^{\circ} \mathrm{C}$ 加热 $45 \mathrm{~h}$ 后, 发现萠分子从胶囊中释放出来, 而 $\mathrm{CDCl}_{3}$ 作为客体被包 裹到分子胶囊中; 如果将等量的焦棓酚杯 [4]芳烃和芘 加入到 $\mathrm{CDCl}_{3}$ 中, $70{ }^{\circ} \mathrm{C}$ 加热 $24 \mathrm{~h}$, 得到只包裹 $\mathrm{CDCl}_{3}$ 的 分子胶囊.

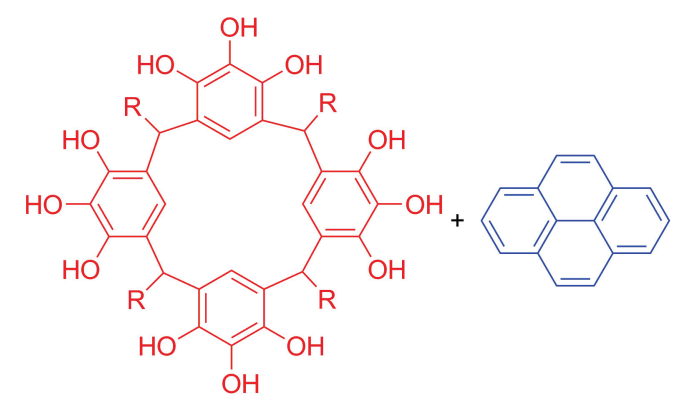

$\mathrm{R}=\mathrm{C}_{10} \mathrm{H}_{21}$

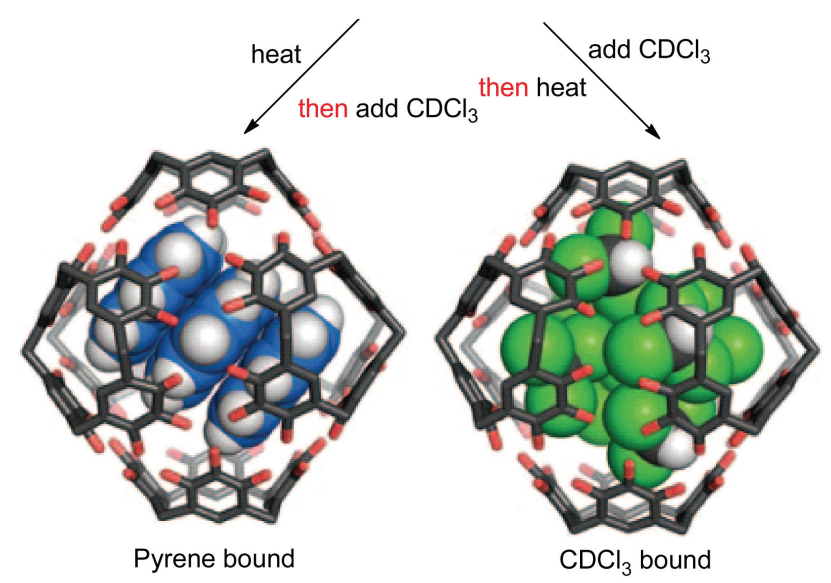

Scheme 7
Rebek 等 ${ }^{[34]}$ 将带有长烷基链的焦棓酚杯 [4]芳烃衍 生物溶解到常见烷烃、带支链的烷烃或环烷烃中，此衍 生物会组装成六聚体的分子胶囊.胶囊中包裹溶剂分 子, 并且所包裹的溶剂分子的数量和其体积大小有关 (Eq. 2).

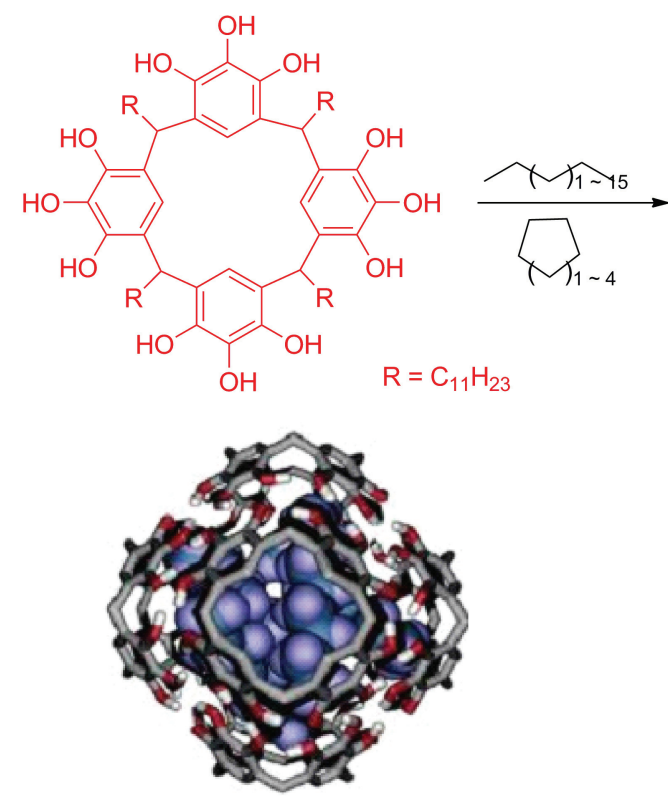

(2)

Cohen 等 ${ }^{[35]}$ 还用此带有长烷基链的焦棓酚杯 [4]芳 烃衍生物组成的六聚体形式分子胶囊作为纳米级微反 应器包裹氯仿和苯分子, 并用核磁共振实验研究客体分 子在胶囊中的状态.

\section{3 雷锁酚形杯芳烃}

雷锁酚形的杯芳烃和焦棓酚形的杯芳烃结构类似， 并且与焦棓酚形的杯芳烃相比其更容易功能化. 因此雷 锁酚形的杯芳烃与其衍生物可利用多种形式(静电作 用、金属配位和氢键等)组装成分子胶囊.

Verboom 等 ${ }^{[36]}$ 制备了 4 个吡啶盐的雷锁酚形杯 [4] 芳烃，并用其和一些一价阴离子(溴离子、硝酸根离子、 醋酸根离子和甲苯磺酸根离子)组装成了一系列新型 $[2+4]$ 的分子胶囊(Eq. 3). 分子胶囊通过“吡啶-阴离子吡啶” 的三元离子作用稳定. 它还可以包裹酚类和苯胺 类客体. 包裹客体后，新的分子胶囊由 “吡定-阴离子吡啶” 和 “吡啶-客体-吡啶” 两种三元离子作用共同稳 定.

Yamanaka 等 ${ }^{[37]}$ 设计合成了带有吡啶基团和酰胺基 团的雷锁酚形杯 [4]芳烃衍生物. 等量的此衍生物和钯 (II)或铂(II)金属能够通过金属配位和氢键作用组装成混 合型的超分子胶囊(Scheme 8). 核磁共振和质谱证实了 此分子胶囊的形成. 此分子胶囊还可以包裹一些外形尺 寸和胶囊内部空腔容积相吻合的客体. 由于客体的存 在，此胶囊的构型在包裹前后是不同的一一客体会使得 


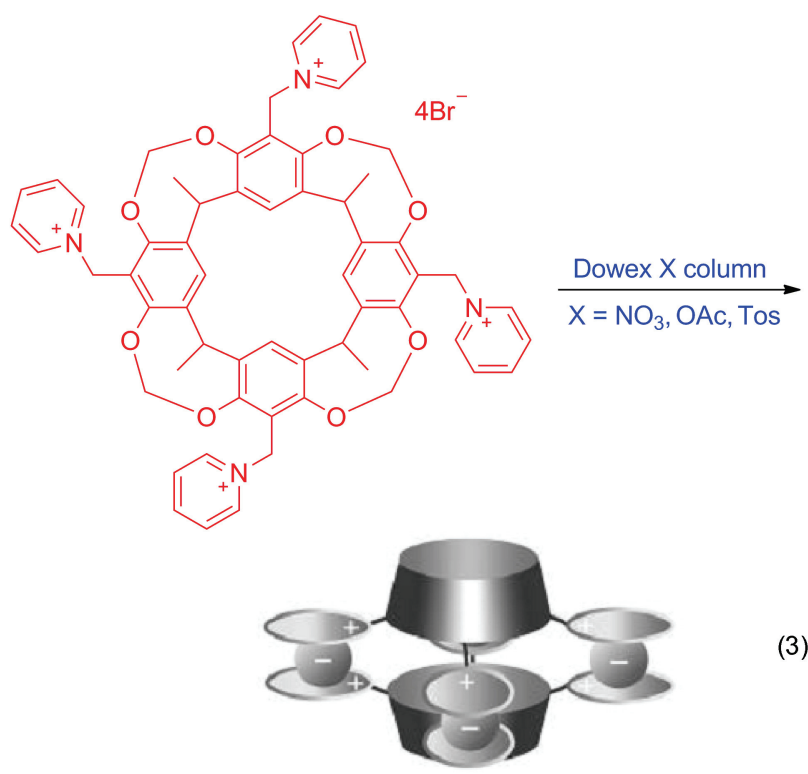

胶囊扭曲. 客体从胶囊的 “进/出” 交换速率受胶囊中的 氢键影响.

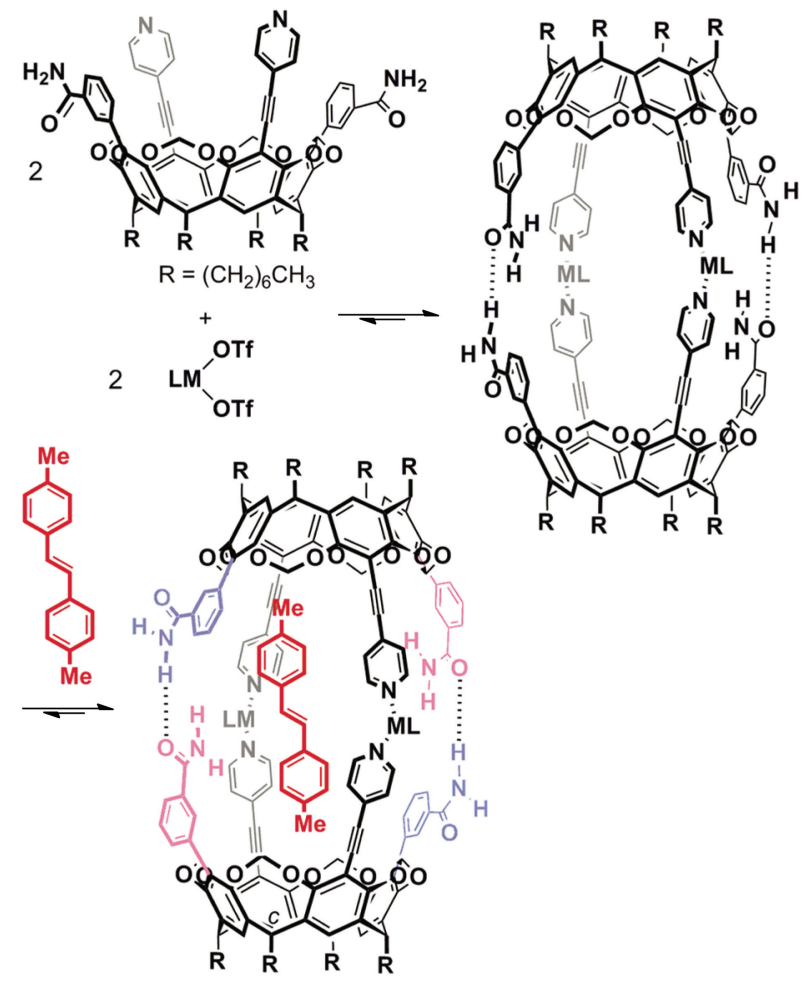

Scheme 8

Paek 等 ${ }^{[38]}$ 用含有苯甲酰肼的雷锁酚形杯 [4]芳烃衍 生物以分子间氢键作用组装分子胶囊(Eq. 4). 此分子胶 囊通过 8 个氢键稳定, 其中四个是两个主体分子对应的 苯甲酰肼基之间的氢键 $(\mathrm{N}-\mathrm{H} \cdots \mathrm{O}=\mathrm{C})$; 另外 4 个来自 于两主体分子内部的氢键 $\left(\mathrm{O}-\mathrm{H}_{2} \mathrm{C}-\mathrm{O} \cdots \mathrm{H}-\mathrm{N}\right)$. 在 $\mathrm{C}_{2} \mathrm{D}_{2} \mathrm{Cl}_{4}$ 溶液中, 此胶囊可以包裹多中客体. 包裹不同 客体的胶囊的稳定性不同: $\mathrm{CH}_{3} \mathrm{SO}_{3}^{-}>\mathrm{CH}_{3} \mathrm{CO}_{2}^{-}>$
$\mathrm{CH}_{3} \mathrm{CH}_{2} \mathrm{NH}_{2} \bullet \mathrm{HCl} \approx \mathrm{CH}_{3} \mathrm{NH}_{2} \bullet \mathrm{HCl}>\left(\mathrm{CH}_{3}\right)_{4} \mathrm{~N}^{+}>$toluene $>$ $\mathrm{C}_{2} \mathrm{D}_{2} \mathrm{Cl}_{4}$.
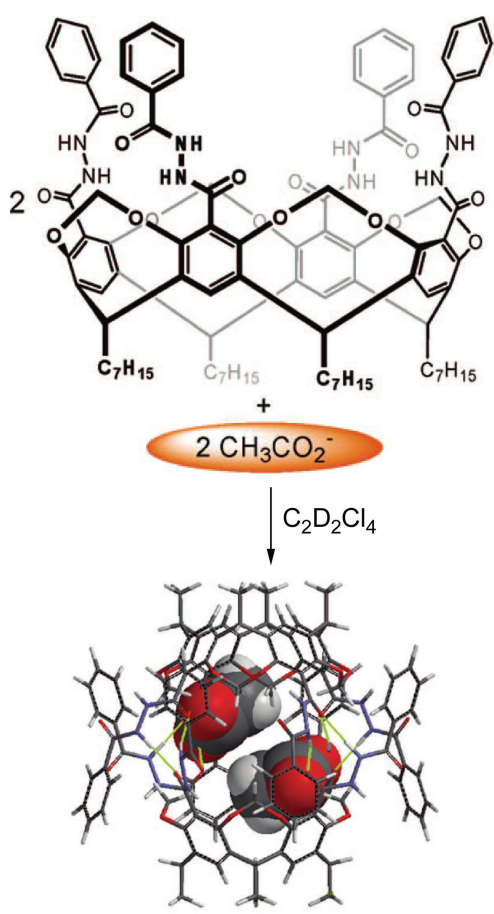

(4)

Rebek 等在利用氢键自组装成分子胶囊方面做了大 量的深入细致的工作. 他们合成了带有强氢键给体和受 体的雷锁酚形杯 [4]芳烃衍生物, 并以此两种衍生物为 构筑模块组装成分子胶囊(Scheme 9).

这两种分子胶囊分别通过酰胺之间的分子间氢键 和硫嫝之间的分子间氢键稳定. 它们有足够大的空腔可 以包裹体积很大的客体, 比如长度极大地支链状烷烃. 最令人惊奇的是，当加入合适的连接臂后(分子 8 和 9 ), 分子胶囊还能扩展(Schemes 10, 11). 扩展后，分子胶囊 的空腔长度最大能增加 $80 \%$ 以上. 更大的空腔赋予了分 子胶囊更丰富的络合性质.

在这些分子胶囊包裹客体分子时有一个有趣的现 象一一分子胶囊对客体分子识别有很大的形状选择性, 即分子胶囊只能络合与其内部空腔形状匹配的客体，如 不匹配则不能络合. 如 Scheme 12 所示, 胶囊 III 是扭曲 的结构，则其只能络合弯曲的客体 $\mathrm{a}$, 而不能包裹直的 客体 $b$; 而直形的分子胶囊 6.88 .6 的情形则正好相反. 以上这些分子胶囊在一些领域有很大的潜在用途, 如: 分子胶囊 6.6 用于羧酸和异氰基的反应的研究 ${ }^{[48]}$ 及用于 增强三氮唑生成的选择性和反应活性等 ${ }^{[49]}$.

以雷锁酚形杯 [4]芳烃还可以构建大空穴分子

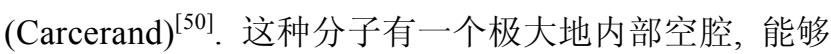
把络合的客体分子完全包裹住，使其即使在很高的温度 下也很难逃逸. 将此大空穴分子适当的衍生化, 就可以 得到极好的构建分子胶囊的模块. 这些模块通过自组装 

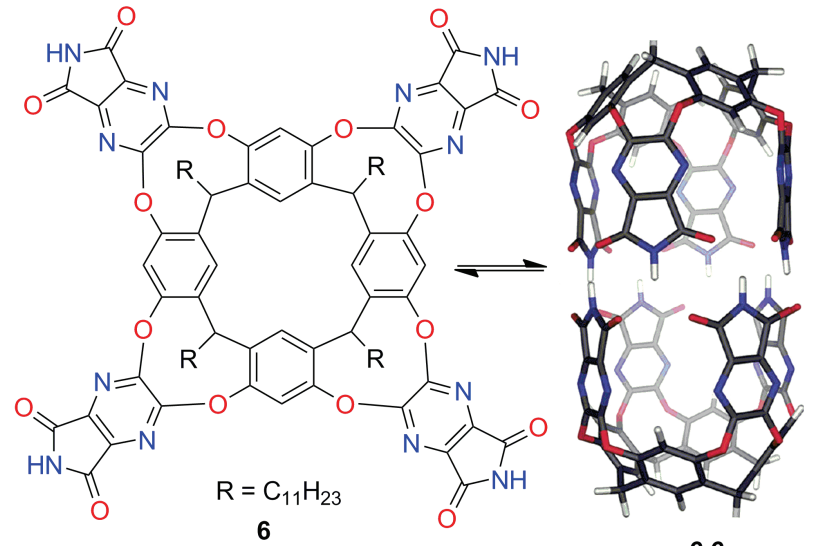

6.6
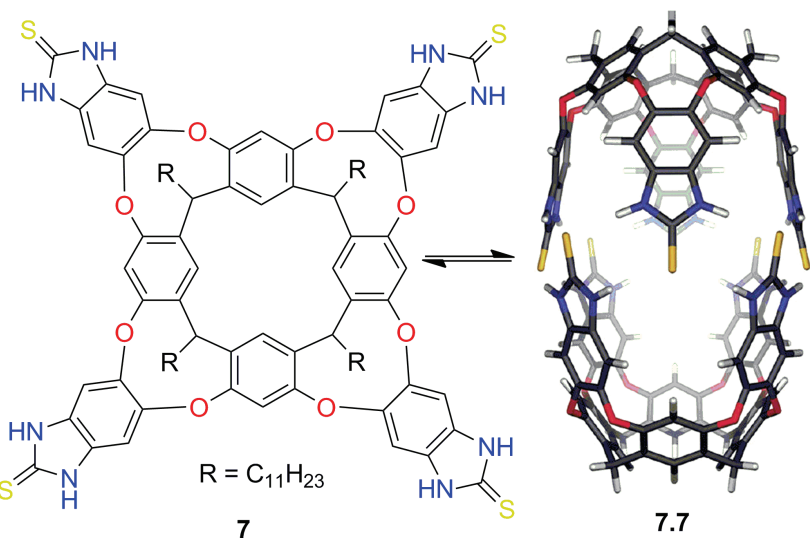

7.7

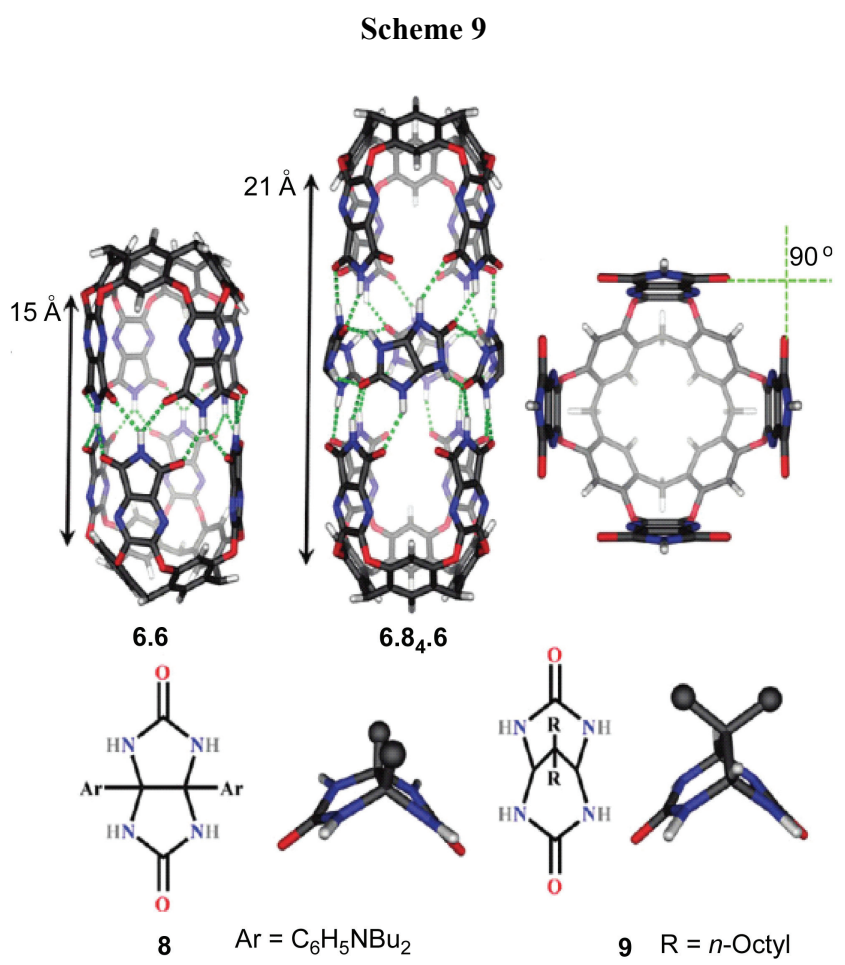

Scheme 10

得到的分子胶囊将拥有更大的内部空间, 其可以包裹更 大的分子, 用途也更加广泛.

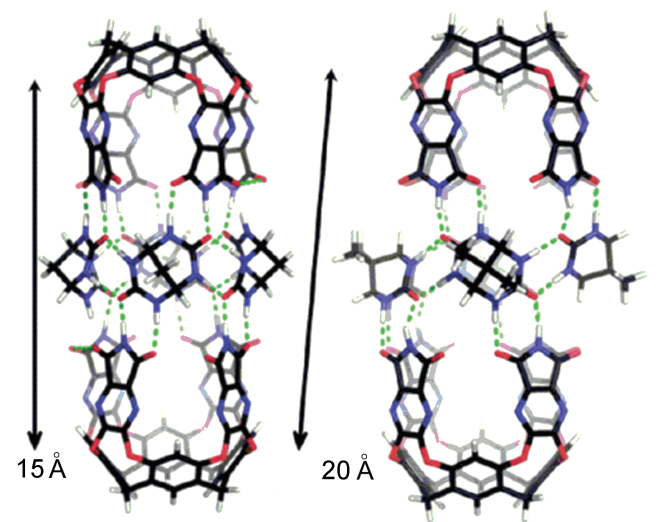

$6.9_{4} .6 \quad 6.9^{\prime} \cdot{ }^{2} \cdot 9_{2} \cdot 6$
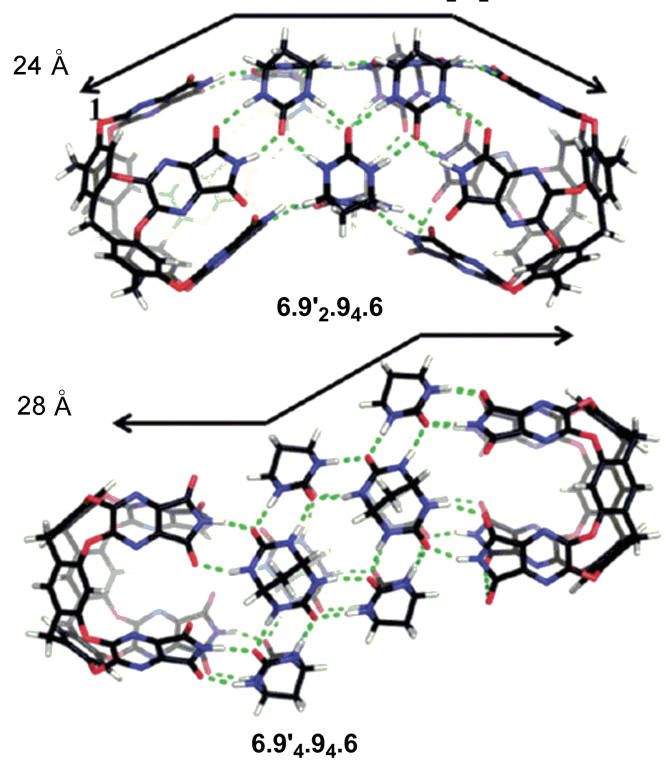

Scheme 11

在这个领域 Ramamurthy 等 $^{[51]}$ 的工作具有代表性. 他们合成了带有 8 个羧基的大空穴分子(Scheme 13). 此 分子可溶于偏碱性的水溶液中, 并且可在水溶液中自组 装成分子胶囊. 因为其有极大空穴，此分子胶囊可以在 溶液中包裹多种客体. Ramamurthy 等用此种分子胶囊 做了很多有价值的工作: 在此分子胶囊里进行可控的单 质氧氧化烯烃的光化反应 ${ }^{[52]}$; 用此胶囊控制对苯二乙 烯的光致变构和苯乙烯的二聚反应 ${ }^{[53]}$ 等.

虽然此种分子胶囊应用较多，但其只局限于碱性的 水溶液中, 因为大空穴分子 $\mathbf{1 0}$ 只溶于碱性水溶液. 如果 要将这类大空穴分子组装的分子胶囊应用于医药领域, 如药物传递等，就必须对其衍生化，使其溶于纯水中， 以适应人体的生理环境. Gibb 等 ${ }^{[54]}$ 完成了这方面的工作 (Scheme 14). 他们在分子 $\mathbf{1 0}$ 的 8 个羧基上反应上带有 羟基末端的聚酯，合成了超枝化的大空穴分子 11. 大量 的着基使得分子 11 能很好的在中性的水中溶解，并使 其具有很好的生物相容性. 此超枝化的分子 11 可以在 中性的水中, 自组装形成分子胶囊. 这种分子胶囊可 


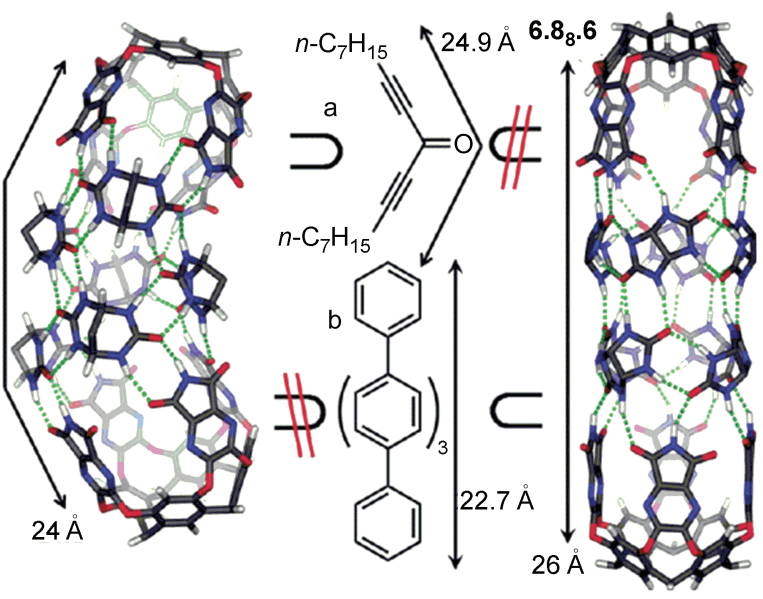

Scheme 12

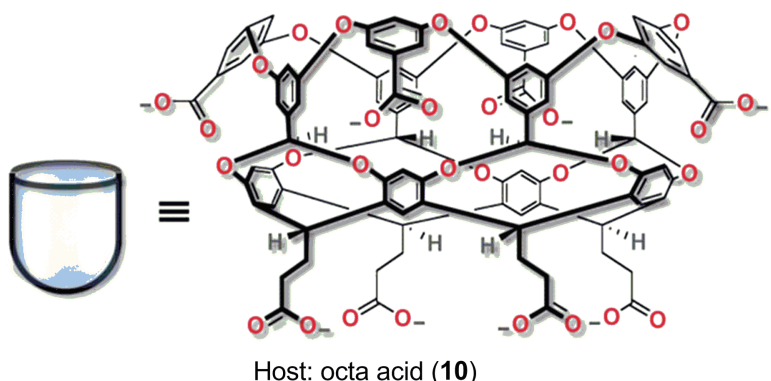

Scheme 13

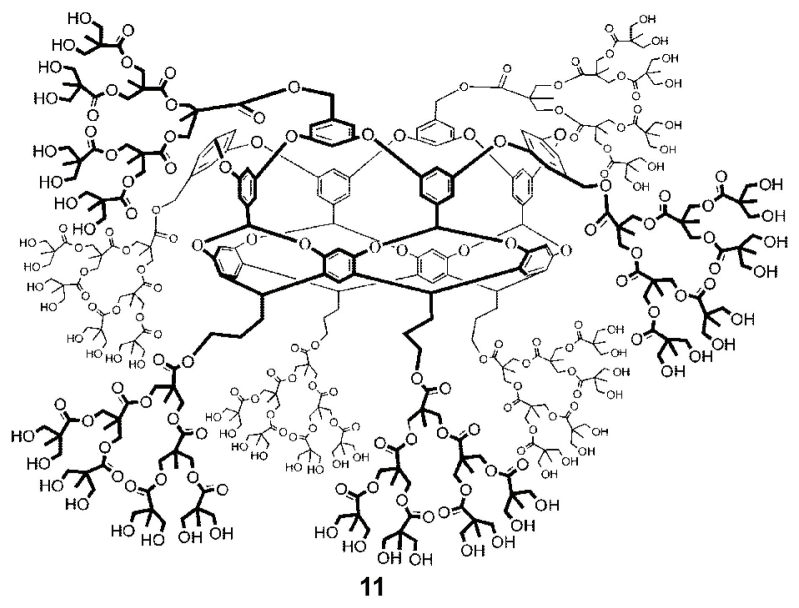

Scheme 14

以在水溶液中包裹多种客体(Eq. 5). 其在生物医药领域 具有较大的应用价值.
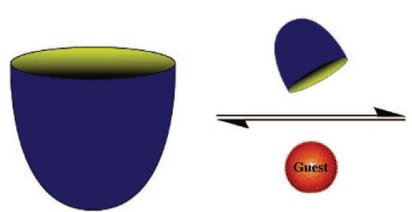

Dendronized cavitand 11

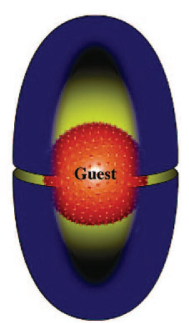

(5)

\section{3 杯吡咯}

在杯吡咯是杯芳烃的类似物, 是由若干个吡咯重复 单元组成的大环, 如: 杯 [4]吡咯是由 4 个吡咯重复单元 组成的大环. 在杯 [4]吡咯的桥基碳原子上共有 8 个取代 基，使其不易被氧化成卟啉. 因其构象与杯 [4]芳烃类 似, 所以被 Sessler 等命名为 “杯吡咯” ${ }^{[29]}$. 杯吡咯具有 圆锥状的立体构型，因此它被广泛的应用于构造分子胶 囊.

Kohnke 等 ${ }^{[5]}$ 合成了在桥基碳上用硝基苯衍生化的 杯 [4]吡咯衍生物 12. 在乙腈溶液中, 12 可组装成分子胶 囊包裹 1,4-对苯二甲酸 ${ }^{[56]}$. 分子胶囊络合物的晶体结构 表明：1,4-对苯二甲酸位于 $\mathbf{1 2}$ 形成的分子胶囊的中心; 客体上的羒酸根与吡咯上的氨基形成氢键，同时客体的 苯环和主体上硝基苯的苯环间存在 “边一面” 的 $\pi-\pi$ 作 用. 通过核磁共振研究发现, 客体分子在胶囊中是可以 绕其中心轴旋转的(Eq. 6).

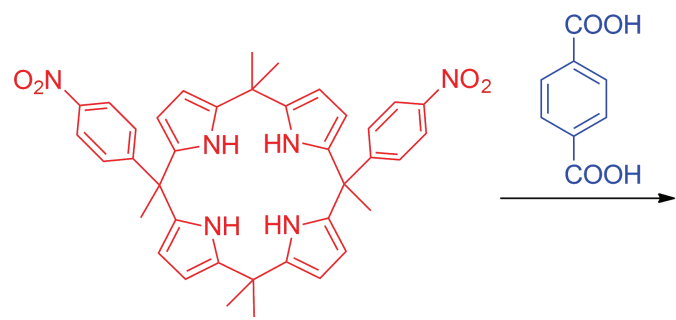

12

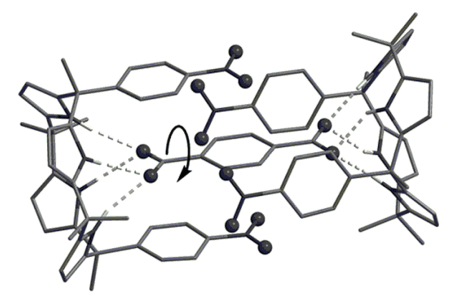

含有艮基的杯吡咯，可通过分子间的氢键自组装成 分子胶囊. Ballester 等 ${ }^{[57 ~ 60]}$ 在这一领域做了大量的工作. 他们在杯 [4]吡咯桥基碳原子上衍生化，合成了含有嫝 基的杯[4]吡咯衍生物 13. 这种化合物可在二氯甲烷中 与客体 14 络合, 从而组装成含有两个主体分子和两个 客体分子的分子胶囊 $\left(1_{2} \subset 13_{2} \text { 如 Eq. } 7 \text { 所示 }\right)^{[57]}$. 除此之 外, 主体 13 还可以在二氯甲烷中与三甲基膦氧化物(客 体分子 15$)$ 组装成类似的分子胶囊 $\left(1_{2} \subset 13_{2}\right)$. 当客体 14 和 15 同时存在于溶液中时，两种客体分子和主体 13 还 可组装成 $14 \cdot 15 \subset 13_{2}$ 形式的分子胶囊，即分子胶囊中同 时包裹两种客体分子. 这几种分子胶囊都是动力学和热 力学稳定的. 其中由客体 14 和主体 13 形成的分子胶囊 $\left(14_{2} \subset 13_{2}\right)$ 更稳定一些. 


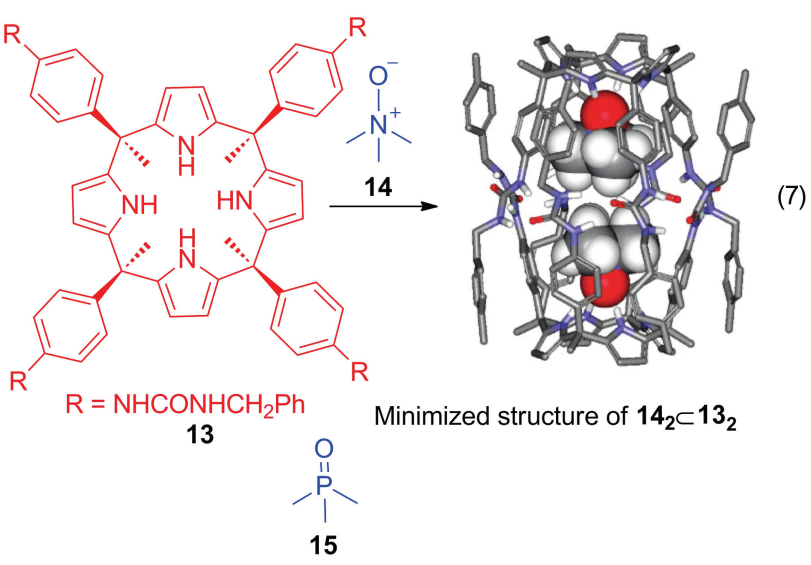

此外, 主体分子 13 还可与客体 $\mathbf{1 6}$ 在二氯甲烷中组 装成分子胶囊 $(16 \subset 13 \cdot 13, E q .8)^{[58]}$. 被包裹的客体分子 16 通过与杯 [4]吡咯中心的两个氢键稳定在分子胶囊中 心. 核磁共振实验证实此结果. 这一分子胶囊同样是高 度的热力学和动力学稳定的.
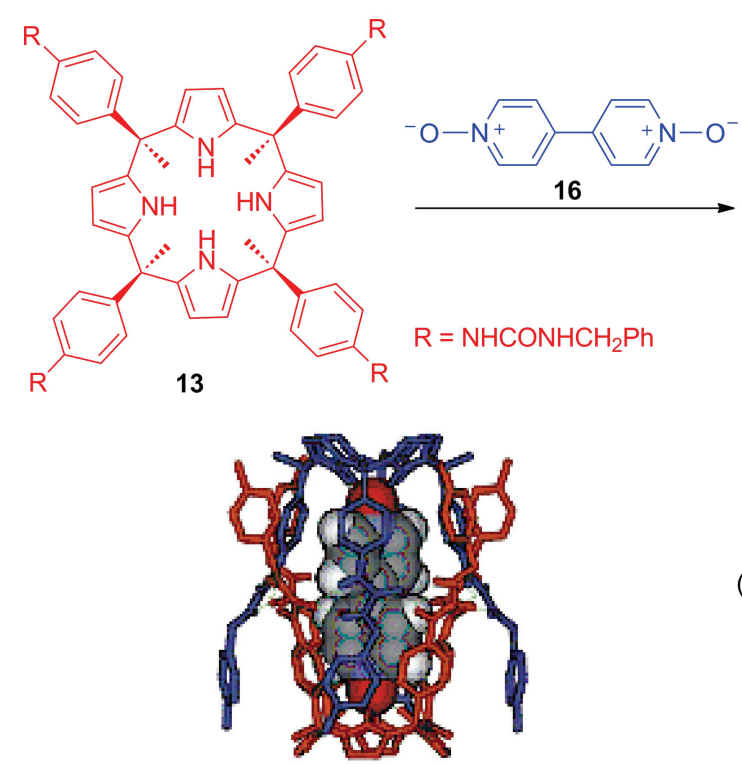

Minimized structure of $16 \subset 13 \cdot 13$

Sessler 等 ${ }^{[61]}$ 利用分子间的电荷转移作用使主体和 客体分子组装成分子胶囊. 他们合成了一种含有四硫富 瓦烯这一富电子基团的杯 [4]吡咯衍生物 17. 首先, 杯 [4]吡咯的衍生物 17 在二氯甲烷中与四丁基氯化铵 (TBACl) 络合 (Scheme 15). 络合的结果使得 17 的构型发 生改变. 其变成能包裹 $\mathrm{C}_{60}$ 的碗状构型. 当缺电子的富 勒烯加入到溶液中后, 其与富电子的四硫富瓦烯基团作 用, 产生电荷转移现象, 溶液的颜色发生改变. 此时, 富勒烯和主体分子 17 络合. Job-plot 结果表明富勒烯和 主体 17 的结合比为 $1: 2$. 络合的结果是客体分子位于 主体分子碗状空腔的内部并贴近四硫富瓦烯基团组装 成分子胶囊.

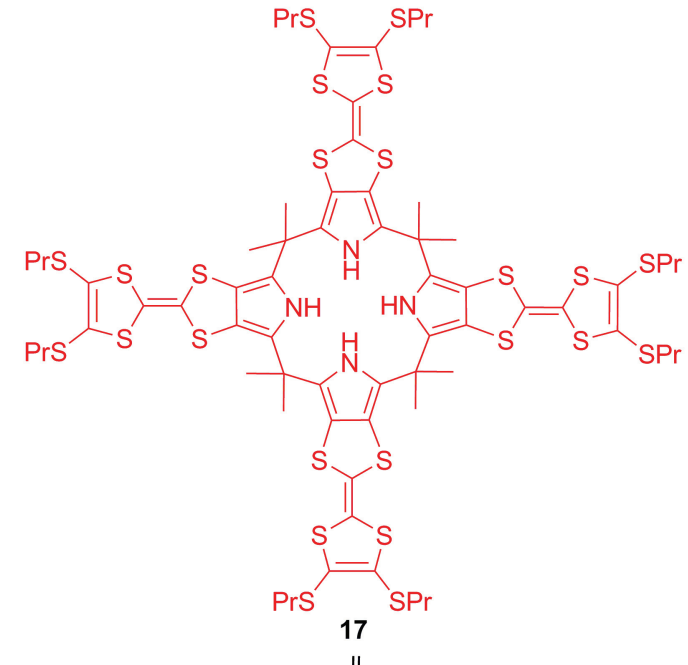
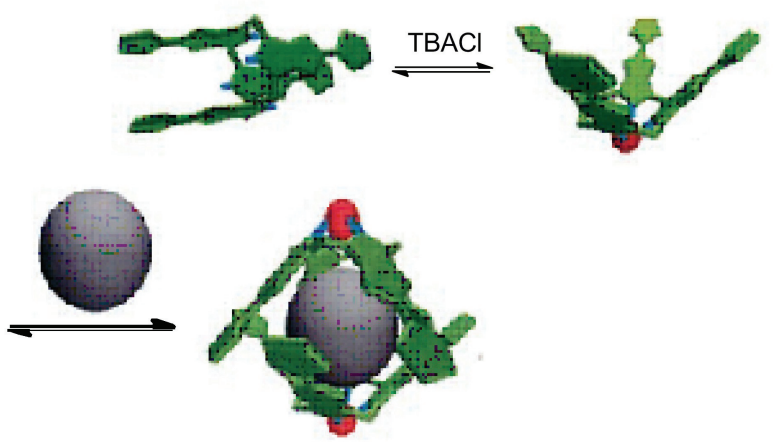

Minimized structure of $\mathrm{C}_{60} \subset\left(\mathrm{Cl}^{-} \bullet 17\right)_{2}$

Scheme 15

\section{4 卟啉}

因其特殊的结构，卟啉可通过氢键、金属离子的协 同作用和卟啉环中心金属原子的协同作用自组装成一

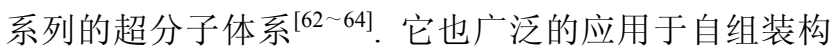
建分子胶囊 ${ }^{[65 ~ 70] . ~}$

Johnston 等 ${ }^{[70]}$ 设计合成了 $\mathrm{V}$ 形的分子 18 . 在金属卟 啉的锌原子和吡啶配体之间的分子间协同作用下，两个 $\mathrm{V}$ 形分子 18 在溶液中组装成分子胶囊(Eq. 9). 此分子胶 囊的形成是溶剂依赖性的——在 DMF, THF，氯仿或苯 溶液中, 分子胶囊的核磁信号变宽很难分辨, 但是在邻 二甲苯、甲苯或二氯甲烷中, 核磁信号变得尖锐很容易 分辨. 造成这一现象的原因可能是溶剂分子充当客体的 能力不同. 此分子胶囊的空腔可以包裹一些体积合适的 客体，如化合物 19. 当客体 19 被包裏在胶囊中时，由于 主体分子的强磁场屏蔽作用，客体分子上的氢原子的核 磁信号向高场大幅移动.

卟啉衍生物也可通过金属配位生成分子胶囊. Ikeda 等 ${ }^{[69]}$ 合成了卟啉的衍生物 20 和 21. 分别用两个衍生物 20 和 21, 与 4 个配体 22 通过金属配位键交联自组装形 成分子胶囊 23 和 24 (Eq. 10). 这两种分子胶囊具有较很 

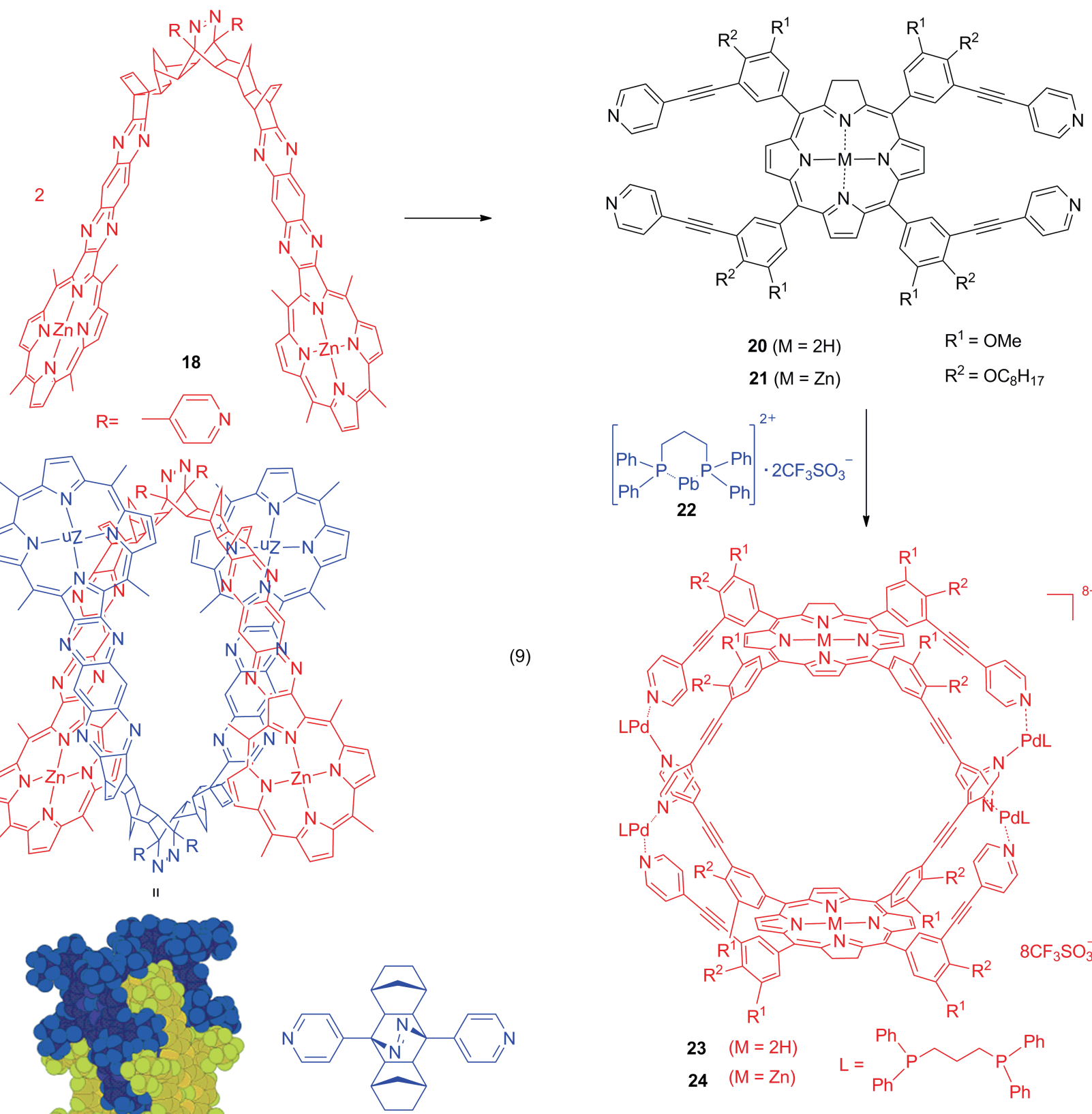

19

(9)

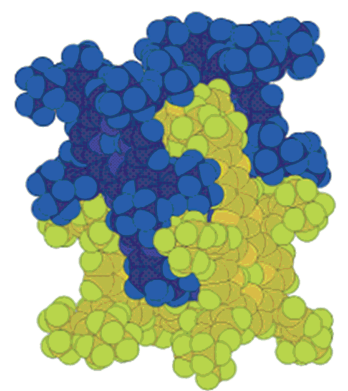

Schematic representation of the capsule (space filling model)

大的空腔, 因此, 它们可以络合体积比较大的客体. 例 如: 分子胶囊 24 可以在氯仿中包裹客体 25, 并且通过 客体分子的吡啶氮原子和金属卟啉上的锌原子配位，使 得客体稳定在胶囊中间.

\section{5 环三藜芦烯(CTV)}

环三荺芦烯 $(\mathrm{CTV})$ 是一个环状的三聚体. 它呈碗状 构型，并拥有一个较浅的分子空腔. 利用其这一特性， 人们用 CTV 或其衍生物构建了多种不同的分子胶囊, 如 Hong 等 ${ }^{[71]}$ 设计合成了两种在上边缘分别带有羧基和 氨基的 CTV 衍生物. 这两种衍生物在 DMSO 中可通过

$$
\begin{array}{ll}
20(M=2 H) & R^{1}=O M e \\
21(M=Z n) & R^{2}=\mathrm{OC}_{8} \mathrm{H}_{17}
\end{array}
$$

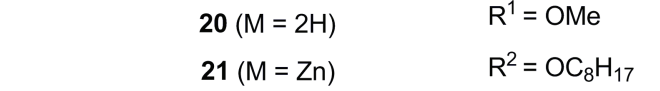

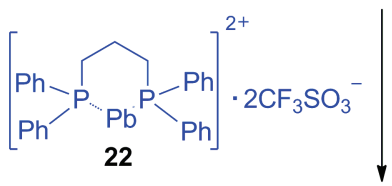

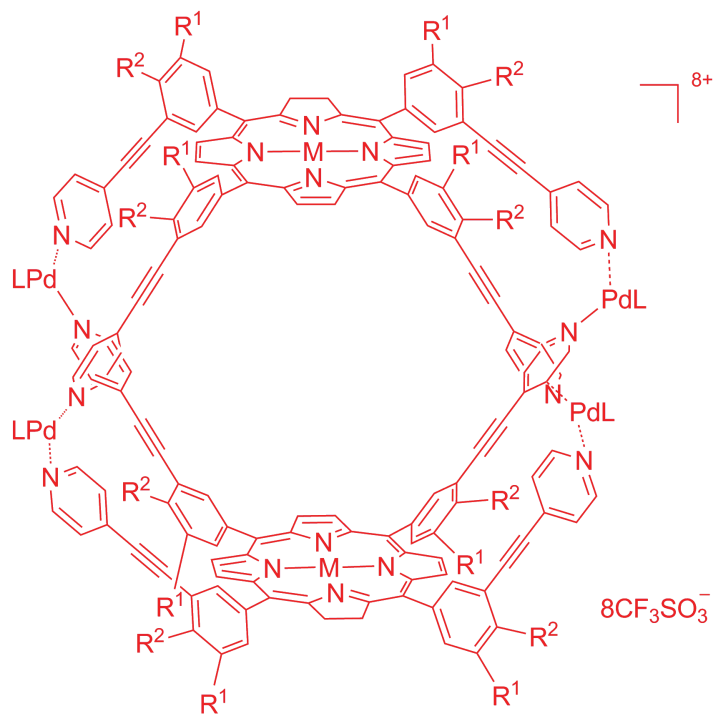

羧基和氨基的间的静电作用自组装成分子胶囊. 这种分 子胶囊可在 DMSO 中包裹多种客体.

Hardie 等 ${ }^{[72]}$ 合成了含有吡定基团的 CTV 衍生物 26. 将化合物 26 溶解到六氟磷酸银的乙腈溶液中, 得到了 以 26 为配体和银离子配位的配合物(Eq. 11). 此配合物 是 $\left[\mathrm{M}_{2} \mathrm{~L}_{2}\right]$ 形式的分子胶囊. 在此分子胶囊中含有两个配 体、两个银离子和两个乙腈分子.

Hardie 等 ${ }^{[73]}$ 还利用金属配位的方式, 将 CTV 的衍 生物 27 组装成分子胶囊. 其将 CTV 衍生物 27 的 $\mathrm{CF}_{3} \mathrm{CH}_{2} \mathrm{OH}$ 溶液加入到 $\mathrm{Ag}\left[\mathrm{Co}\left(\mathrm{C}_{2} \mathrm{~B}_{9} \mathrm{H}_{11}\right)_{2}\right]$ 的乙腈溶液中, 

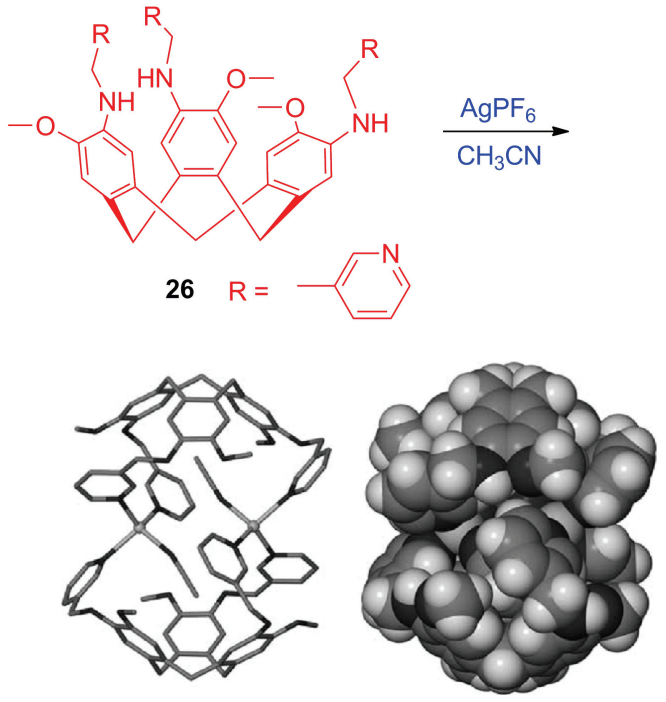

控制乙腈和 $\mathrm{CF}_{3} \mathrm{CH}_{2} \mathrm{OH}$ 的体积比为 $3: 7$. 将此混合液 敞口放置几天得到橘黄色的晶体. 经过解析, 晶体为 $\mathrm{CTV}$ 衍生物 27 组成的分子胶囊. 分子胶囊中包含 2 个 27 分子、 1 个银离子、 1 个水分和 1 个乙腈分子(Eq. 12).

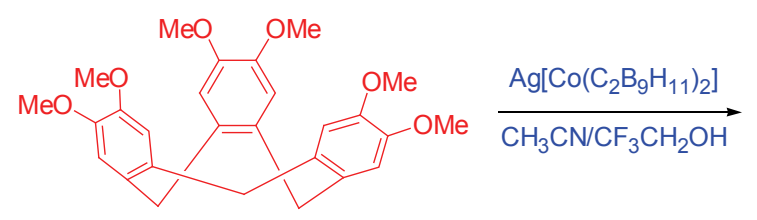

27

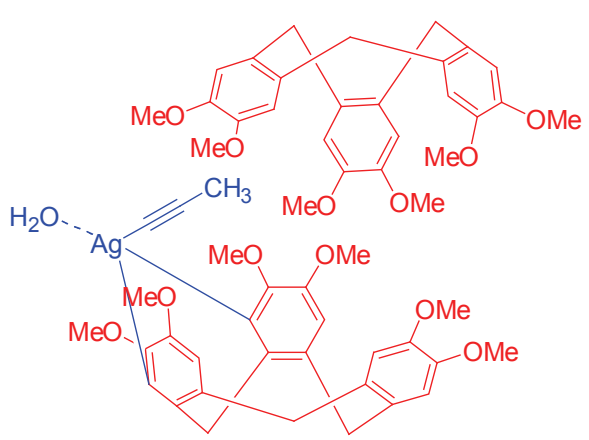

Kubo 等 ${ }^{[74]}$ 还利用 CTV 和含有嗍酸的杯芳烃类似物 通过离子对作用组装成分子胶囊, 并通过 $\mathrm{pH}$ 值来调节 组装的过程.

\section{6 葫芦腿}

葫芦嫝 $[n](n=5 \sim 10)$ 是由甘腿重复单元通过亚甲 基桥连形成的环状聚合物. 它拥有一个桶状的外形, 内 有较大的空腔, 并且在上下口两端分布有羰基, 是一种 极好的穴状主体, 被广泛应用于主一客体络合和超分子 自组装. 将葫芦腿和金属离子组装可以得到各色各样的 分子胶囊 ${ }^{[75 ~ 80]}$.

Long 等 ${ }^{[81]}$ 用葫芦腿 $[5](\mathrm{Q}[5])$ 和镧系金属离子以不 同的方法组装成的分子胶囊可以包裹硝基离子或氯离
子(Scheme 16). 在分子胶囊 $\mathbf{2 8}$ 中, 八配位的 $\mathrm{La}(\mathrm{III})$ 金属 离子位于胶囊的中轴位置. 其中 1 个和 5 个葫芦脲 [5]的 末端氧原子及 3 个水分子配位; 另一个 $\mathrm{La}(\mathrm{III})$ 金属离子 和 5 个葫芦腿[5]的末端氧原子、 2 个水分子及 1 个单齿 的硝基分子配位; 硝基离子被包裹在分子胶囊中; 分子 胶囊 29 的结构与 $\mathbf{2 8}$ 的类似, 只不过氯离子被包裹到分 子胶囊中, 且其中一个 $\mathrm{La}(\mathrm{III})$ 金属离子没有与一个双齿 的硝基离子配位.

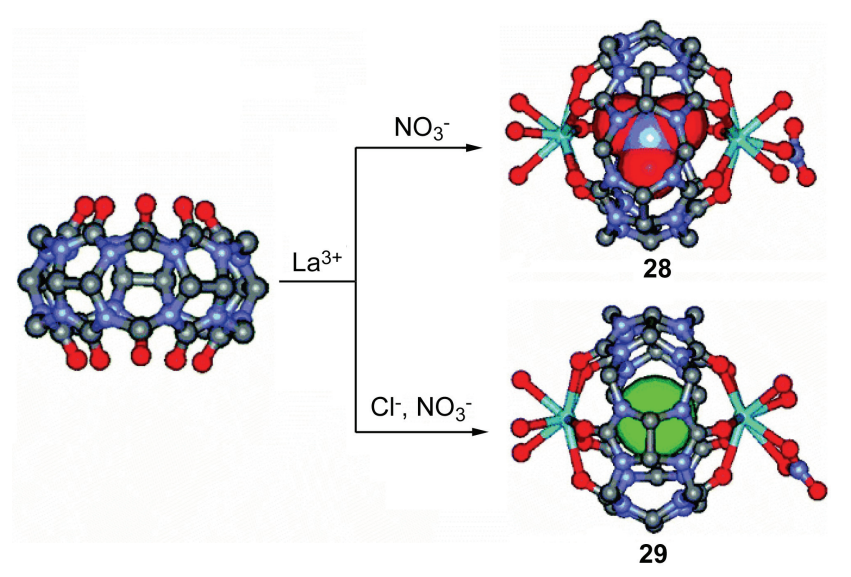

Scheme 16

Zhang 等 ${ }^{[82]}$ 用 3 种不同葫芦腿[5] (Scheme 17)与不 同金属离子组装成多种分子胶囊. 比如 Scheme 18 所示 的由双甲基化的葫芦腿[5]与钝金属离子组装成的分子 胶囊. 在此分子胶囊中, 两个钝金属离子以不同的配位 方式连接到双甲基化葫芦腿[5]的两端(DMeQ[5]), 并且 氯离子被包裹在分子胶囊的内部. 其中十配位的 $\operatorname{Gd}(1)$ 完全封住了 $\mathrm{DMeQ}[5]$ 的一个开口, 它与 3 个羰基氧 $[\mathrm{O}(1), \mathrm{O}(2), \mathrm{O}(3), \mathrm{O}(4)$ 和 $\mathrm{O}(5)], 3$ 个水分子 $[\mathrm{O}(2 \mathrm{~W})$, $\mathrm{O}(3 \mathrm{~W})$ 和 $\mathrm{O}(5 \mathrm{~W})$ 及被包裹的氯离子 $\mathrm{C}(11)$ ]配位; 另一个 八配位的 $\operatorname{Gd}(2)$ 封住了 $\mathrm{DMeQ}[5]$ 另一端的部分开口, 它 与两个羰基氧 $[\mathrm{O}(8)$ 和 $\mathrm{O}(9)]$ 及 6 个水分子 $[\mathrm{O}(1 \mathrm{~W})$, $\mathrm{O}(4 \mathrm{~W}), \mathrm{O}(6 \mathrm{~W}), \mathrm{O}(7 \mathrm{~W}), \mathrm{O}(9 \mathrm{~W})$ 和 $\mathrm{O}(14 \mathrm{~W})$ ]配位.

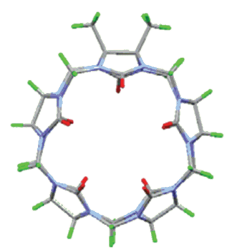

a

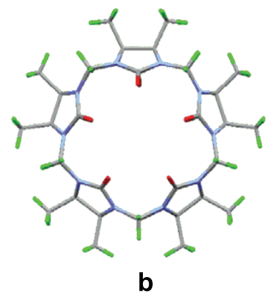

Scheme 17

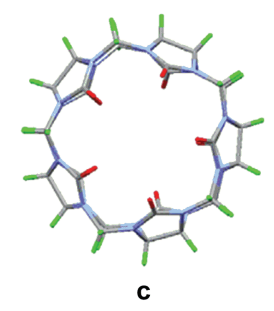

c
这些分子胶囊并没有直接相连, 但是它们通过水分 子和水分子之间的氢键或氯离子与水分子之间的氢键 (Scheme 18 中 b 和 $\mathrm{c}$ 所示)连接起来形成一维的超分子链 (Scheme 18 中 d 所示). 

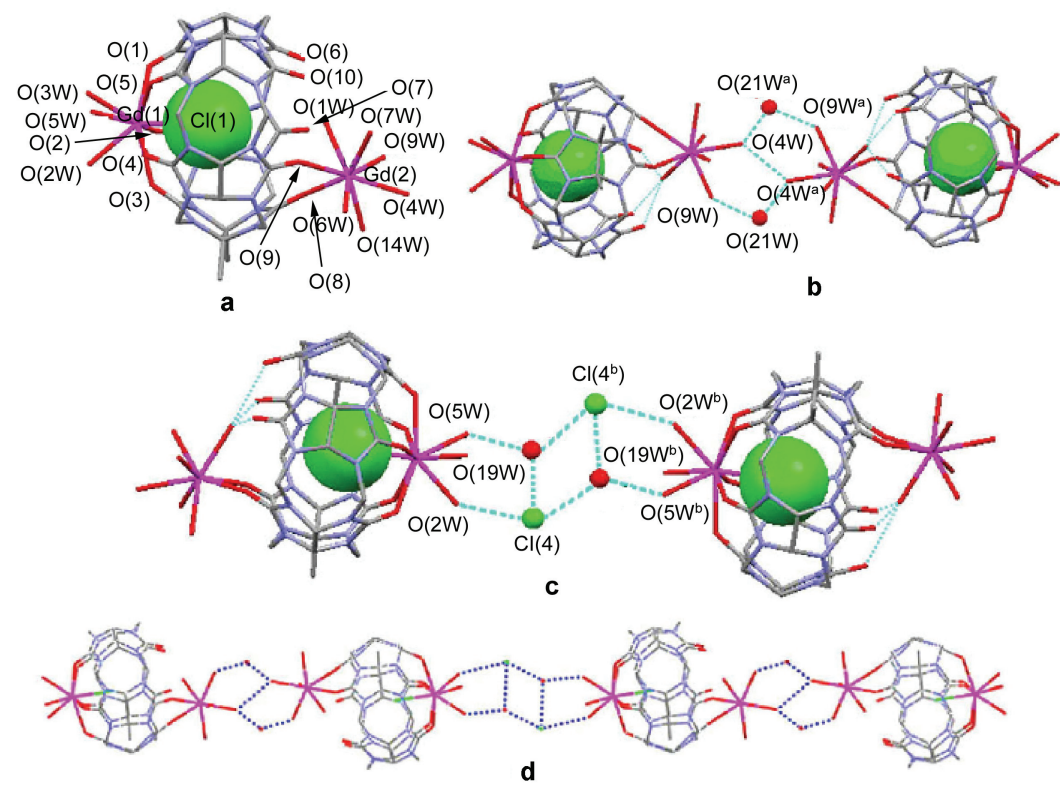

Scheme 18

Mohanty 等 ${ }^{[83]}$ 利用葫芦脲[7] $\left(\mathrm{CB}_{7}\right)$ 、硫磺素 $\mathrm{T}(\mathrm{ThT})$ 和金属离子在水溶液中组成有极强苂光的分子胶囊 (Scheme 19). 向 1:1 ( $\left.\mathrm{CB}_{7} \supset \mathrm{ThT}\right)$ 的 $\mathrm{ThT}$ 和 $\mathrm{CB}_{7}$ 复合物 溶液中, 加入与 $\mathrm{ThT}$ 竞争的客体, 即金属离子, 使得 ThT 的苂光强度显著下降. 然而, 向 $2: 1\left(\mathrm{CB}_{7} \supset \mathrm{ThT}\right)$ 的 $\mathrm{ThT}$ 和 $\mathrm{CB}_{7}$ 形成的复合物中, 加入金属离子会使得 ThT 的苂光强度极大地增强(加入 $\mathrm{Ca}^{2+}$ 荧光增强约 270 倍; 加入 $\mathrm{Na}^{+}$苂光增强约 160 倍). $2: 1$ ( $\mathrm{CB}_{7} \supset \mathrm{ThT}$ )的络合方 式为金属离子的提供了较强的负电荷密度, 使得金属离 子把 $2: 1\left(\mathrm{CB}_{7} \supset \mathrm{ThT}\right)$ 的络合物分组, 并把 $\mathrm{CB}_{7}$ 密封, 形 成分子胶囊. 这保护了被 $\mathrm{CB}_{7}$ 包裹的 $\mathrm{ThT}$ 客体分子, 使 其苂光强度增强.

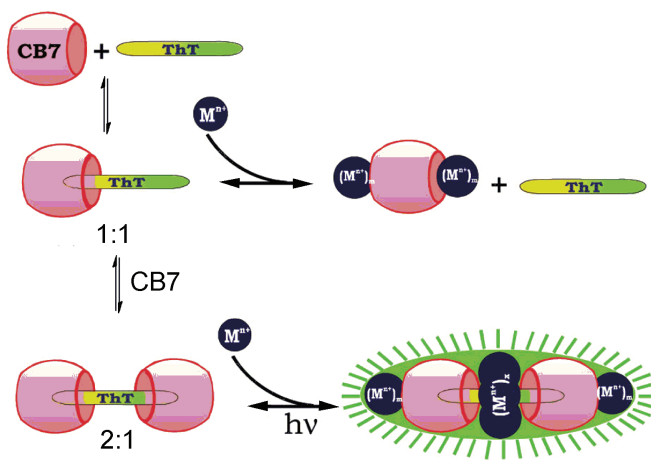

Scheme 19

如果再向形成的分子胶囊溶液中, 加入强竞争性的 客体 1-金刚烷胺盐酸盐, 会分解分子胶囊使 ThT 释放出 来. 因此，此分子胶囊可用于药物的络合和传递、菼光 开关或作为基础模块构建具有独特性质的超分子体系.

\section{7 结论}

分子胶囊可由多种主体分子构建，但是常见的大环 主体应用最为广泛，因为人们对它们的研究由来已久, 且深入透彻, 对它们各方面的性质了解深入. 因此, 本 文综述了用常见的大环主体通过超分子自组装构建分 子胶囊 ${ }^{[84 ~ 88]}$. 组装的驱动力是分子间的弱相互作用力: 氢键、静电作用、疏水作用、 $\pi-\pi$ 堆积作用和范德华力 等甚至更强的金属配位作用. 自组装形成的分子胶囊可 以包裹合适的客体分子一一客体分子体积和相应的分 子胶囊内部空间容积相匹配，或者是客体分子的外表面 和胶囊的内表面 “互补” 。被包裹的客体分子被分子胶 囊形成的机械 “壁垒” 从其周围环境中隔离出来. 这使 得客体分子具有许多被包裹前不具备的性质. 因此，分 子胶囊可以用作微容器、微反应器、分子传感器和分子 逻辑门，还可用于载药及药物的控制释放和化学反应的 催化等.

但是在此领域，还有一些困难需要克服. 其中分子 胶囊的表征还存在一些问题. 核磁共振成像虽能提供一 些信息，但不能直观的表征；质谱技术只能提供分子聚 集体的信息，并不能给出分子胶囊形成; $X$ 射线晶体衍 射虽然能提供分子胶囊形成的直接证据，但其只对晶体 有效, 对溶液中形成的较松散的分子胶囊就无能为力; 电镜技术只能观测到较大分子胶囊，对尺寸较小的就力 不从心. 因此, 要全面详细的表征各种分子胶囊需要一 些更好的检测技术．再者，有关分子胶囊的所有技术都 还只是概念性的研究，还属于浅显的基础研究阶段. 其 实用化的道路还很长. 


\section{References}

[1] Conn, M. M.; Rebek, J. Jr. Chem. Rev. 1997, 97,1647.

[2] Ong, W.; Gómez-Kaifer, M.; Kaifer, A. E. Org. Lett. 2002, 4, 1791.

[3] Liu, M.; Li, S.; Zhang, M.; Zhou, Q. Z.; Wang, F.; Hu, M.; Fronczek, F. R.; Li, N.; Huang, F. Org. Biomol. Chem. 2009, 7, 1288.

[4] Wang, F.; Zheng, B.; Zhu, K.; Zhou, Q.; Zhai, C.; Li, S.; Li, N.; Huang, F. Chem. Commun. 2009, 4375.

[5] Yu, G.; Zhang, Z. B.; Han, C.; Xue, M.; Zhou, Q. Z.; Huang, F. H. Chem. Commun. 2012, 48, 2958.

[6] Yan, X. Z.; Wei, P. F.; Xia, B. Y.; Huang, F. H.; Zhou, Q. Z. Chem. Commun. 2012,48, 4968.

[7] Shi, J.; Chen, Y.; Wang, Q.; Liu, Y. Adv. Mater. 2010, $22,1$.

[8] Cram, D. G. Science 1983, 219, 1177.

[9] Yamanaka, M.; Rebek, J. Jr. Chem. Commun. 2004, 1690.

[10] Körner, S. K.; Tucci, F. C.; Rudkevich, D. M.; Heinz, T.; Rebek, J. Jr. Chem.-Eur. J. $\mathbf{2 0 0 0}, 6,187$.

[11] Ziegler, M.; Brumaghim, J. L.; Raymond, K. N. Angew. Chem., Int. Ed. 2000, 39, 4119.

[12] Yoshizawa, M.; Kusukawa, T.; Fujita, M.; Yamaguchi, Y. J. Am. Chem. Soc. 2000, 122, 6311.

[13] Boris Breiner, B.; Clegg, J. K.; Nitschke, J. R. Chem. Sci. 2011, 2, 51.

[14] Rebek, J. Jr. Angew. Chem., Int. Ed. 2005, 44, 2068.

[15] Fiedler, D.; Leung, D. H.; Bergman, R. G.; Raymond, K. N. Acc. Chem. Res. 2005, 38, 349.

[16] Yoshizawa, M.; Klosterman, J. K.; Fujita, M. Angew. Chem., Int. Ed. 2009, 48, 3418 .

[17] Pluth, M. D.; Bergman, R. G.; Raymond, K. N. Acc. Chem. Res. 2009, 42, 1650

[18] Brunsveld, L.; Folmer, B. J.; Meijer, E. W.; Sijbesma, R. P. Chem. Rev. 2001, 101, 4071.

[19] Prins, L. J.; Reinhoudt, D. N.; Timmerman, P. Angew. Chem., Int. Ed. 2001, 40,2382.

[20] Bong, D. T.; Clark, T. D.; Granja, J. R.; Ghadiri, M. R. Angew. Chem., Int. Ed. 2001, 40, 988.

[21] Balzani, V.; Cred, A.; Raymo, F. M.; Stoddart, J. F. Angew. Chem., Int. Ed. 2000, 39, 3348.

[22] Whitesides, G. M.; Boncheva, M. Proc. Natl. Acad. Sci. U. S. A. 2002, 99, 4769.

[23] Clark, T. D.; Tien, J.; Duffy, D. C.; Paul, K. E.; Whitesides, G. M.; J. Am. Chem. Soc. 2001, 123, 7677.

[24] Pease, A. R.; Jeppesen, J. O.; Stoddart, J. F.; Luo, Y.; Collier, C. P.; Heath, J. R. Acc. Chem. Res. 2001, 34, 433.

[25] Hamelin, B.; Jullien, L.; Derouet, C.; Penhoat, C. H.; Berthault, P. J. Am. Chem. Soc. 1998, 120, 8438 .

[26] Bakirci, H.; Nau, W. M. J. Org. Chem. 2005, 70, 4506.

[27] Anczewski, W.; Dodziuk, H.; Ejchart, A. Chirality, 2003, 15, 654.

[28] Toro-Sánchez, C. L. D.; Ayala-Zavala, J. F.; Machi, L.; Santacruz, H.; Villegas-Ochoa, M. A.; Alvarez-Parrilla, E.; González-Aguilar, G. A. J. Incl. Phenom. Macrocycl. Chem. 2010, 67, 431.

[29] Gale, P. A.; Anzenbacher, P.; Sessler, J. L. Coord. Chem. Rev. 2001, 222, 57.

[30] Dalgarno, S. J.; Raston, C. L. Dalton Trans. 2003, 287.

[31] Atwood, J. L.; Barbour, L. J.; Hardie, M. J.; Raston, C. L.; Stattonand, M. N.; Webb, H. R. CrystEngComm 2001, 4, 1.

[32] Hardie, M. J.; Makha, M.; Raston, C. L. Chem. Commun. 1999, 2409.

[33] Kvasnica, M.; Chapin, J. C.; Purse, B. W. Angew. Chem., Int. Ed. 2011, 50, 2244.

[34] Palmer, L. C.; Rebek, J. Jr. Org. Lett. 2005, 7, 5.
[35] Avram, L.; Cohen, Y. Org. Lett. 2006, 8, 2.

[36] Oshovsky, G. V.; Reinhoudt, D. N.; Verboom, W. J. Am. Chem. Soc. 2006, 128, 5270.

[37] Yamanaka, M.; Kawaharada, M.; Nito, Y.; Takaya, H.; Kobayashi, K. J. Am. Chem. Soc.2011, 133, 16650.

[38] Park, Y. S.; Paek, K. Org. Lett. 2008, 10, 21.

[39] Heinz, T.; Rudkevich, D. M.; Rebek, J. Nature 1998, 394, 764.

[40] Rebek, J. Angew. Chem., Int. Ed. 2005, 44, 2068.

[41] Jeong, K. S.; Tjivikua, T.; Rebek, J. Jr. J. Am. Chem. Soc. 1990, 112,3215

[42] Ajami, D.; Rebek, J. J. Am. Chem. Soc. 2006, 128, 5314.

[43] Ajami, D.; Rebek, J. Angew. Chem., Int. Ed. 2007, 46, 9283.

[44] Moon, K.; Chen, W. Z.; Ren, T.; Kaifer, A. E. CrystEngComm 2003, 5, 451 .

[45] Piacenza, G.; Beguet, C.; Wimmer, E.; Gallo, R.; Giorgi, M. S. C. Cryst. Struct. Commun. 1997, 53, 1459.

[46] Shivanyuk, A.; Rebek, J. J. Am. Chem. Soc. 2002, 124, 12074.

[47] Ajami, D.; Rebek, J. Jr. Nat. Chem. 2009, 1, 87.

[48] Hou, J. L.; Ajami, D.; Rebek, J. Jr. J. Am. Chem. Soc. 2008, 130, 7810.

[49] Cantillo, D.; Avalos, M.; Babiano, R.; Cintas, P.; Jiménez, J.; Palacios, J. C. Org. Biomol. Chem. 2011, 9, 7638.

[50] Cram, D. J.; Karbach, S.; Kim, Y. H.; Baczynskyj, L.; Kallemeyn, G. W. J. Am. Chem. Soc.1985, 107, 2575.

[51] Chen, J. Y. C.; Jayaraj, N.; Jockusch, S.; Ottaviani, M. F.; Ramamurthy, V.; Turro, N. J. Am. Chem. Soc. 2008, 130, 7206.

[52] Natarajan, A.; Kaanumalle, L. S.; Jockusch, S.; Gibb, C. L. D.; Gibb, B. C.; Turro, N. J.; Ramamurthy, V. J. Am. Chem. Soc. 2007, 129, 4132 .

[53] Parthasarathy, A.; Kaanumalle, L. S.; Ramamurthy, V. Org. Lett. 2007, 9, 24.

[54] Giles, M. D.; Liu, S.; Emanuel, R. L.; Gibb, B.; Grayson, S. M. J. Am. Chem. Soc. 2008, 130, 14430.

[55] Bruno, G.; Cafeo, G.; Kohnke, F. H.; Nicol, F. Tetrahedron 2007, 63, 10003.

[56] Cafeo, G.; Kohnke, F. H.; Valenti; White, A. J. P. Chem. Eur. J. 2008, 14, 11593.

[57] Gil-Ramìre, G.; Chas, M.; Balleste, P. J. Am. Chem. Soc. 2010, 132, 2520.

[58] Ballester, P.; Gil-Ramírez, G. Proc. Natl. Acad. Sci. U. S. A. 2009, 106,10455 .

[59] Chas, M.; Gil-Ramírez, G.; Ballester, P. Org. Lett. 2011, 13, 13.

[60] Chas, M.; Gil-Ramírez, G.; Escudero-Adán, E.; Benet-Buchholz, J.; Ballester, P. Org. Lett. 2010, 12, 8.

[61] Nielsen, K. A.; Cho, W. S.; Sarova, G. H.; Peterse, B. M.; Bond, A. D.; Becher, J.; Jensen, F.; Guldi, D. M.; Sessler, J. L.; Jeppesen, J. O. Angew. Chem., Int. Ed. 2006, 45, 6848.

[62] Wyler, R.; Mendoza, J. D.; Rebek, J. Jr. Angew. Chem., Int. Ed. Engl. 1993, 32, 1699.

[63] Kuroda, Y.; Kawashima, A.; Hayashi, Y.; Ogoshi, H. J. Am. Chem. Soc.1997, 119, 4929.

[64] Schenning, A. P. H. J.; Benneker, F.; Geurts, H. P.; Liu, X. Y.; Nolte, R. J. M. J. Am. Chem. Soc. 1996, 118, 8549.

[65] Nakazawa, J.; Hagiwara, J.; Mizuki, M.; Shimazaki, Y.; Tani, F.; Naruta, Y. Angew. Chem., Int. Ed. 2005, 44, 3744.

[66] Johnston, M. R.; Lyons, D. M. Taylor Francis 2005, 1061.

[67] Reek, J. N. H.; Schenning, A. P. H. J.; Bosman, A. W.; Meijer, E. W.; Crossley, M. J. Chem. Commun. 1998, 11.

[68] Nakazawa, J.; Mizuki, M.; Shimazaki, Y.; Tani, F.; Naruta, Y. Org. Lett. 2006, 8, 19.

[69] Ikeda, A.; Ayabe, M.; Shinkai, S.; Sakamoto, S.; Yamaguchi, K. Org. Lett. 2000, 2, 23. 
[70] Johnston, M. R.; Latter, M. J.; Warrener, R. N. Org. Lett. 2002, 4, 13.

[71] Lee, S. B.; Hong, J. I. Tetrahedron Lett. 1996, 37, 8501.

[72] Sumby, C. J.; Hardie, M. J. Angew. Chem., Int. Ed. 2005, 44, 6395.

[73] Ahmad, R.; Hardie, M. J. Cryst. Growth Des. 2003, 3, 493.

[74] Kataoka, K.; James, T. D.; Kubo, Y. J. Am. Chem. Soc. 2007, 129, 15126.

[75] Zhang, Y. Q.; Zhu, Q. J.; Xue, S. F.; Tao, Z. Molecules 2007, 12, 1325.

[76] Thuéry, P. Cryst. Growth Des. 2009, 9, 2.

[77] Liu, J. X.; Long, L. S.; Huang, R. B.; Zheng, L. S. Cryst. Growth Des. 2006, 6, 11.

[78] Liu, J.; Gu, Y. F.; Lin, R.; Yao, W.; Liu, X.; Zhu, J. Supramol. Chem. 2010, 22, 130.

[79] Xiao, X.; Chen, K.; Xue, S. F.; Zhu, Q. J.; Tao, Z.; Wei, G. J. Mol. Struct. 2010, 969, 216.
[80] Kushwaha, S.; Rao, S. A.; Sudhakar, P. P. Inorg. Chem. 2012, 51, 267.

[81] Liu, J. X.; Long, L .S.; Huang, R. B.; Zheng, L. S. Inorg. Chem. 2000, 46, 24

[82] Zhang, Y. Q.; Zeng, J. P.; Zhu, Q. J.; Xue, S. F.; Tao, Z. J. Mol. Struct. 2009, 929, 167.

[83] Choudhury, S. D.; Mohanty, J.; Pal, H.; Bhasikuttan, A. C. J. Am. Chem. Soc. 2010, 132, 1395.

[84] Ajami, D.; Rebek, J. Jr. Acc. Chem. Res. 2012, DOI: 10.1021/ar300038r.

[85] Arunachalam, M.; Ghosh, P. Chem. Commun. 2011, 47, 8477.

[86] Rebek, J., Jr. Chem. Commun. 2007, 2777.

[87] Rieth, S.; Hermann, K.; Wang, B. Y.; Badjic, J. D. Chem. Soc. Rev. 2011, 40, 1609.

[88] Conn, M. M.; Rebek, J. Jr. Chem. Rev. 1997, 97, 1647. 